\title{
Neuerungen in der Qualitätssicherung im Praxislabor per 1. Januar 2009
}

Beat Bumbacher, ${ }^{a}$ Heini Haldi ${ }^{b}$, Ernst Gähler ${ }^{c}$

a Leiter Tarifdienst der FMH

b Laborbeauftragter KHM

c Vizepräsident und Mitglied des Zentralvorstands der FMH, Leiter Ressort Tarife und Verträge
Korrespondenz:

Dr. med., RA. lic. iur. Beat Bumbacher Tarifdienst FMH

Gösgenstrasse 8

CH-4600 Olten

Tel. 0622879696

Fax 0622879690

beat.bumbacher@fmh.ch

www.fmh.ch
Momentan revidiert das das Bundesamt für Gesundheit (BAG) die Analysenliste (AL), und trotz grosser allgemeiner Unzufriedenheit über die bisher vom BAG publizierten Vorschläge für die erneuerte Liste (Beta- und Gammaversion) weiss die Ärzteschaft nicht, ob ihre eigenen Vorschläge vom BAG aufgenommen werden. Obwohl wir hier nicht von der AL sprechen, ist die Bedeutung dieser Revision für das Praxislabor gross. Mit der Revision wird mehr oder weniger entschieden, ob das Labor mit seiner Präsenzdiagnostik in den Arztpraxen noch eine Zukunft hat. Es sei in diesem Zusammenhang nur daran erinnert, dass bei 88,5\% der Patienten Laboruntersuchungen in der Grundversorgerpraxis durchgeführt werden [1].

Ab 2009 werden im Bereich der Qualitätssicherung im Laborbereich zwei Neuerungen eingeführt, die zwar keinen massiven Einfluss auf die bisherige Laborroutine im Praxisumfeld haben werden, aber trotzdem zur Kenntnis genommen werden sollten. Mit den Fragen zur Qualitätssicherung im medizinischen Labor befasst sich die Schweizerische Kommission für Qualitätssicherung im medizinischen Labor (QUALAB), die aus Vertretern der Leistungserbringer- und Kostenträgerverbände zusammengesetzt ist. Eines der Hauptanliegen zur Gründung der QUALAB war, dass die Ausführung der Gesetzesbestimmungen betreffend Qualitätssicherung durch die Fachleute selbst in die Hand genommen und nicht den Behörden überlassen werden, was nach Art. 58 Abs. 2 KVG und Art. 77 KVV auch möglich wäre. In diesem Sinne wurde die QUALAB schon vor Jahren aktiv und hat ein Konzept erarbeitet, in dem die Voraussetzungen für den Laborbetrieb und die Elemente der Qualitätssicherung festgehalten sind.

\section{Kriterien zum Betreiben von medizinisch-analytischen Laboratorien}

Zur Sicherstellung der Qualität im Labor wird vom Grundsatz ausgegangen, dass gleiche Analysen unabhängig vom Ort der Erbringung gleiche Qualitätskriterien zu erfüllen haben. Die QUALAB hat deshalb bereits im Jahr 1999 die von der Schweizerischen Union für Labormedi- zin (SULM) ausgearbeiteten «Kriterien zum Betreiben von medizinisch-analytischen Laboratorien» (KBMAL) in ihr Konzept übernommen. Die KBMAL unterscheidet in ihren Regelungen zwischen einem Auftrags- (Grosslabor) und einem Praxislabor. Entsprechend findet sich der praktizierende Arzt in den Formulierungen der KBMAL nur schlecht wieder. Um ihm den Nachvollzug dieser verbindlichen Richtlinien zu vereinfachen, wurde von der QUALAB eine Checkliste erstellt, die in der ersten Hälfte des laufenden Jahres eine breite Vernehmlassung in den Dach- und Fachgesellschaften durchlaufen musste. Diese Checkliste soll die Leistungserbringer schrittweise zur Erfüllung der international geltenden Kriterien zum Betreiben von medizinischen Laboratorien führen. Es wird also nicht Neues eingeführt, sondern die seit Jahren geltenden und einzuhaltenden Bestimmungen werden in konkreter Form aufbereitet, um für den Praxislaborverantwortlichen verständlicher zu werden. Sie finden diese Checkliste KBMAL im Internet (www.qualab.ch) oder können sie auch bei Ihrem Qualitätskontrollzentrum (QKZ) anfordern.

\section{Interne Qualitätskontrolle}

Die eigentliche Qualitätskontrolle findet im Laborbereich mittels der obligatorischen externen Ringversuche und der internen Qualitätskontrolle statt. Auch diese beiden Elemente sind im Konzept der QUALAB verankert und haben schon seit Jahren Gültigkeit. Beide Formen der Qualitätskontrolle sind obligatorisch. Es hat sich gezeigt, dass die interne Qualitätskontrolle im Konzept nur ungenügend beschrieben wurde. Während einer sehr langen Zeit wurde deshalb an den Bestimmungen hierfür gefeilt und verhandelt; beteiligt daran waren wiederum alle Interessenverbände sowie Mitglieder der Fachgesellschaften sowie der QKZ. Auch hier folgte parallel zur KBMAL eine breite Vernehmlassung, aus der erst die nun vorliegende Endfassung der Richtlinie als «Interne Qualitätskontrolle» (IQK) entstand. Diese Richtlinie dient als Anleitung zur Durchführung und Interpretation der gesetzlich, vertraglich und im Konzept verlangten internen Qualitätskontrolle im Praxisalltag der Laborana- 
lytik. Die IQK stellt keine neuen oder zusätzlichen Anforderungen, sondern fasst die ab dem neuen Jahr geltenden Bestimmungen für das Praxislabor auf eineinhalb Seiten zusammen. Dabei wurde der Situation des Praxislabors speziell Beachtung geschenkt. Die Häufigkeit der internen Qualitätskontrollen wurde von bisher zweimal täglich auf neu einmal pro zwei Wochen reduziert. Ausgenommen von internen Qualitätskontrollen sind folgende Analysen der Grundversorgerliste: Schwangerschaftstest, StrepASchnelltest, Uricult, Urinteststreifen, Differentialblutbild, Urinsediment. Wichtig zu wissen ist noch, dass die Messwerte der aufgrund der Richtlinie IQK erstellten Kontrollen während fünf Jahren aufbewahrt werden müssen. Die IQK finden Sie ebenfalls auf der Homepage der QUALAB.

\section{Überprüfung der Einhaltung der KBMAL und der IQK}

Die Einhaltung der in der Checkliste geforderten Punkte in der KBMAL ist Sache des Laborbetreibers. Er soll damit in Selbstverantwortung die notwendigen Kriterien in seinem (Praxis-)Labor überprüfen können und diese gegebenenfalls anpassen. Die Überprüfung, ob die Richtlinie IQK eingehalten wird, ist nicht weiter geregelt; dies müsste im Rahmen einer Aktualisierung des QUALAB-Konzeptes erfolgen. Vorerst wird auch hier auf die Selbstverantwortung verwiesen.

\section{Literatur}

1 Beeler I, Szucs T, Gutzwiller F. Ist das Praxislabor medizinisch und wirtschaftlich sinnvoll? Schweizerische Praxislaborstudie 2000. Praxis. 2001;90(20):887-96 